\title{
Fabrication of ultra-thin Si nanopillar arrays for polarization-independent spectral filters in the near-IR
}

Ryan C. Ng, Julia R. Greer, Katherine T. Fountaine

Ryan C. Ng, Julia R. Greer, Katherine T. Fountaine, "Fabrication of ultra-thin Si nanopillar arrays for polarization-independent spectral filters in the nearIR," Proc. SPIE 10541, Photonic and Phononic Properties of Engineered Nanostructures VIII, 105411D (21 February 2018); doi: 10.1117/12.2286804 


\title{
Fabrication of Ultra-Thin Si Nanopillar Arrays for Polarization- Independent Spectral Filters in the Near-IR
}

\author{
Ryan C. Ng*a Julia R. Greer ${ }^{\mathrm{b}}$, Katherine T. Fountaine ${ }^{\mathrm{c}}$ \\ ${ }^{a}$ Division of Chemistry and Chemical Engineering, California Institute of Technology, Pasadena, CA \\ 91125, USA; ${ }^{\mathrm{b}}$ Division of Engineering and Applied Science, California Institute of Technology, \\ Pasadena, CA, 91125, USA; ${ }^{\mathrm{C}}$ NG Next, Northrop Grumman Corporation, One Space Park, Redondo \\ Beach, CA 90278
}

\begin{abstract}
Sub-wavelength arrays have garnered significant interest for many potential optoelectronics applications. We fabricated sub-wavelength silicon nanopillar arrays with a ratio of radius, $r$ and a center-to-center distance, $a$, of $r / a \approx 0.2$ that were fully embedded in $\mathrm{SiO}_{2}$ for narrow stopband filters that are compact and straightforward to fabricate compared to conventional Bragg stack reflectors. These arrays are well-suited for hyperspectral filtering applications in the infrared. They are ultra-thin $(<0.1 \lambda)$, polarization-independent, and attain greater efficiencies enabled by low loss compared to plasmonic-based designs. The choice of $\mathrm{Si}$ as the nanopillar material stems from its low cost, high index of refraction, and a band gap of $1.1 \mathrm{eV}$ near the edge of the visible.

These arrays exhibit narrow near-unity reflectivity resonances that arise from coupling of an incident wave into a leaky waveguide mode via a grating vector that is subsequently reradiated, also known as guided mode resonances (GMRs). Simulations reveal reflectivities of $>99 \%$ with full width at half maxima (FWHM) of $\approx 0.01 \mu \mathrm{m}$. We demonstrate a fabrication route for obtaining nanopillar arrays that exhibit these GMRs. We experimentally observed a GMR with an amplitude of $\sim 0.8$ for filter arrays fabricated on silicon on insulator (SOI) substrates, combined with Fabry-Perot interference that stems from the underlying silicon layer.
\end{abstract}

Keywords: nanopillar array, guided mode resonance, spectral filters

\section{INTRODUCTION}

Spectral filters have a wide range of sensing applications ranging from environmental (hazardous waste, oil, etc) to surveillance. ${ }^{[1-10]}$ In sensing, detectors are sensitive to anywhere from several to hundreds of electromagnetic bands. Based on the number of bands and bandwidth, these systems are separated into multispectral and hyperspectral imaging systems with the former capturing under 10 bands and the latter - hundreds to thousands of bands of narrow width (around 10-20 nm) that allow for a continuous measurement across a full spectrum.

Subwavelength dielectric nanopillar arrays have potential for such spectral filtering applications. In these arrays, rapid spectral variations in reflectivity and transmission are observed. Incident light couples via a grating vector to a leaky waveguide mode that propagates perpendicular to the surface. The subwavelength nature of these arrays only allows the zeroth diffraction order to propagate and transmit through the array. All higher diffraction orders are trapped within the array and eventually become evanescent and couple to leaky modes, which leads to the emergence of sharp near-unity reflectivity resonances. ${ }^{[11,12]}$ This phenomenon is known as a guided mode resonance (GMR).

We fabricated ultra-thin dielectric nanopillar arrays in silicon in the near-infrared with pillar heights $<0.1 \lambda$ that exhibit polarization independence and greater efficiencies due to low loss relative to that of plasmonic-based designs. Silicon is chosen as the dielectric material due to its low cost, high index of refraction, and its band gap that ranges from around 1.1 to $1.7 \mathrm{eV}$ (crystalline versus amorphous silicon) which lies near the edge of the visible regime. 


\section{TUNABILITY OF REFLECTIVITY RESONANCE}

The wavelength position of the GMR in a nanopillar array is controlled through parametric variation of the radius, $r$, periodicity, $a$, and height, $h$. This tunability is demonstrated in Figure 1, determined using finite-difference time-domain simulations. Due to asymmetry of the Fano shape of the resonance, it is difficult to define a background. We quantify the band width with a full-width half-max (FWHM) defined to be the width at half of the peak amplitude, regardless of the background. As shown in Figure 1b, increasing $r$ from $160 \mathrm{~nm}$ to $250 \mathrm{~nm}(a=1050 \mathrm{~nm} ; h=210 \mathrm{~nm})$ results in a slight red-shift moving the peak location from $1526 \mathrm{~nm}$ to $1561 \mathrm{~nm}$, broadening of the resonance with an increase in the FWHM from $3 \mathrm{~nm}$ to $24 \mathrm{~nm}$, and an increase in amplitude of the GMR to near unity. As shown in Figure 1c, increasing $h$ from 80 to $120 \mathrm{~nm}(r=210 \mathrm{~nm} ; a=1050 \mathrm{~nm})$ causes slight red-shifts from $1533 \mathrm{~nm}$ to $1548 \mathrm{~nm}$ with a small increase in band width with FWHM increasing from $7 \mathrm{~nm}$ to $15 \mathrm{~nm}$; the peak amplitude also increases with increasing pillar height. As shown in Figure 1d, small increases in $a$ show the greatest ability to red-shift the location of the resonance out of the three parameters here, while also narrowing and very slightly decreasing the amplitude of the peak. Increasing $a$ from $950 \mathrm{~nm}$ to $1100 \mathrm{~nm}$ shifts the GMR from $1435 \mathrm{~nm}$ to $1626 \mathrm{~nm}$ with a decrease in FWHM from $38 \mathrm{~nm}$ to $19 \mathrm{~nm}$ while maintaining a near unity amplitude. The ability to change these resonance characteristics with easily controlled fabrication parameters creates a large design space, ideal for their utilization in a filter system. Through careful optimization of successive arrays, near-unity contiguous narrow spectral bands can be fabricated that exhibit reflectivity resonances across the NIR allowing their incorporation as notch filters in a hyperspectral imaging system.

(a)
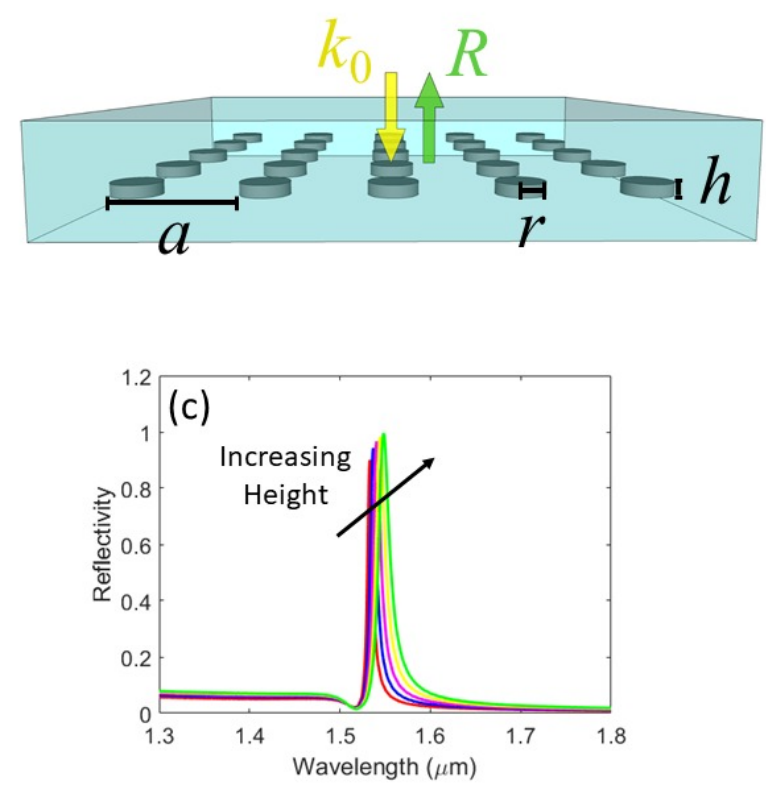
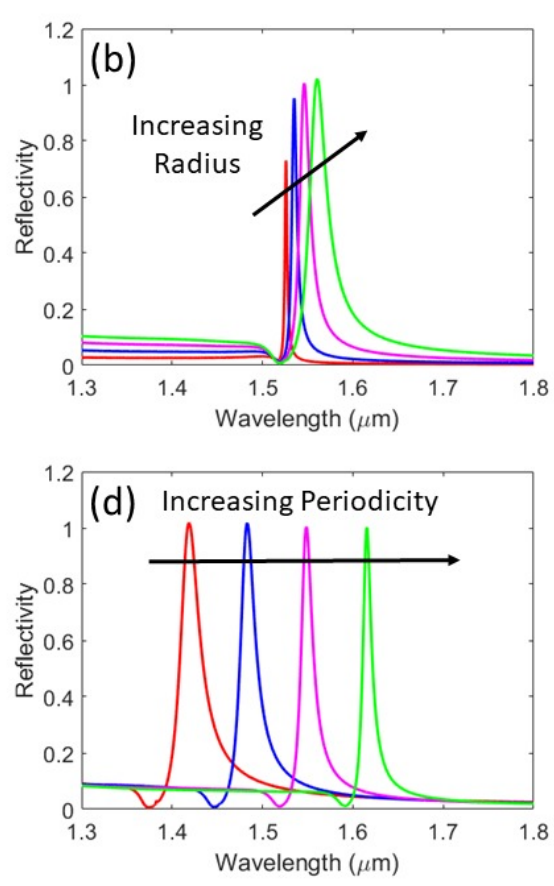

Figure 1. FDTD simulations demonstrating tunability of nanopillar array. (a) Schematic of the nanopillar array indicating the incident and reflected ray and the pillar parameters: period, $a$, radius, $r$, and height, $h$. (b) Varying $r$ from $160 \mathrm{~nm}$ to $250 \mathrm{~nm}$ in increments of $30 \mathrm{~nm}, a=1050 \mathrm{~nm}, h=100 \mathrm{~nm}$ (c) Varying $h$ from $80 \mathrm{~nm}$ to $120 \mathrm{~nm}$ in increments of $10 \mathrm{~nm}, r$ $=210 \mathrm{~nm}, a=1050 \mathrm{~nm}$ (d) Varying $a$ from $950 \mathrm{~nm}$ to $1100 \mathrm{~nm}$ in increments of $50 \mathrm{~nm}, r=210 \mathrm{~nm}, h=100 \mathrm{~nm}$. 


\section{ARRAY FABRICATION}

A schematic illustrating the fabrication process and microscope image of the pillars are shown in Figure 2. We start the process with a silicon-on-insulator (SOI) substrate with a $220 \mathrm{~nm}$ thick device layer, $3 \mu \mathrm{m}$ thick oxide layer, and $675 \mu \mathrm{m}$ thick substrate. The final pillar height is determined via the thickness of the device layer. First, the device layer is thermally oxidized and etched in buffered HF down to the desired pillar height. This surface is patterned in e-beam lithography with a $5 \mathrm{nA}$ beam current and a $610 \mu \mathrm{C} / \mathrm{cm}^{2}$ dosage with $200 \mathrm{~nm}$ thick MaN-2403 negative tone resist which serves as an etch mask. The pattern is then transferred through the entire device layer down to the oxide layer by inductively coupled plasma reactive-ion etching (ICP-RIE) with a pseudo-Bosch etch. ${ }^{[13]}$ Following etching, the resist etch mask is removed in an oxygen plasma. Finally, to maintain no index contrast between the substrate and the cover, $500 \mathrm{~nm}$ of a methylsiloxane spin-on glass (Filmtronics) optimized for gap-filling is applied to the surface to fully encapsulate the nanowire array.

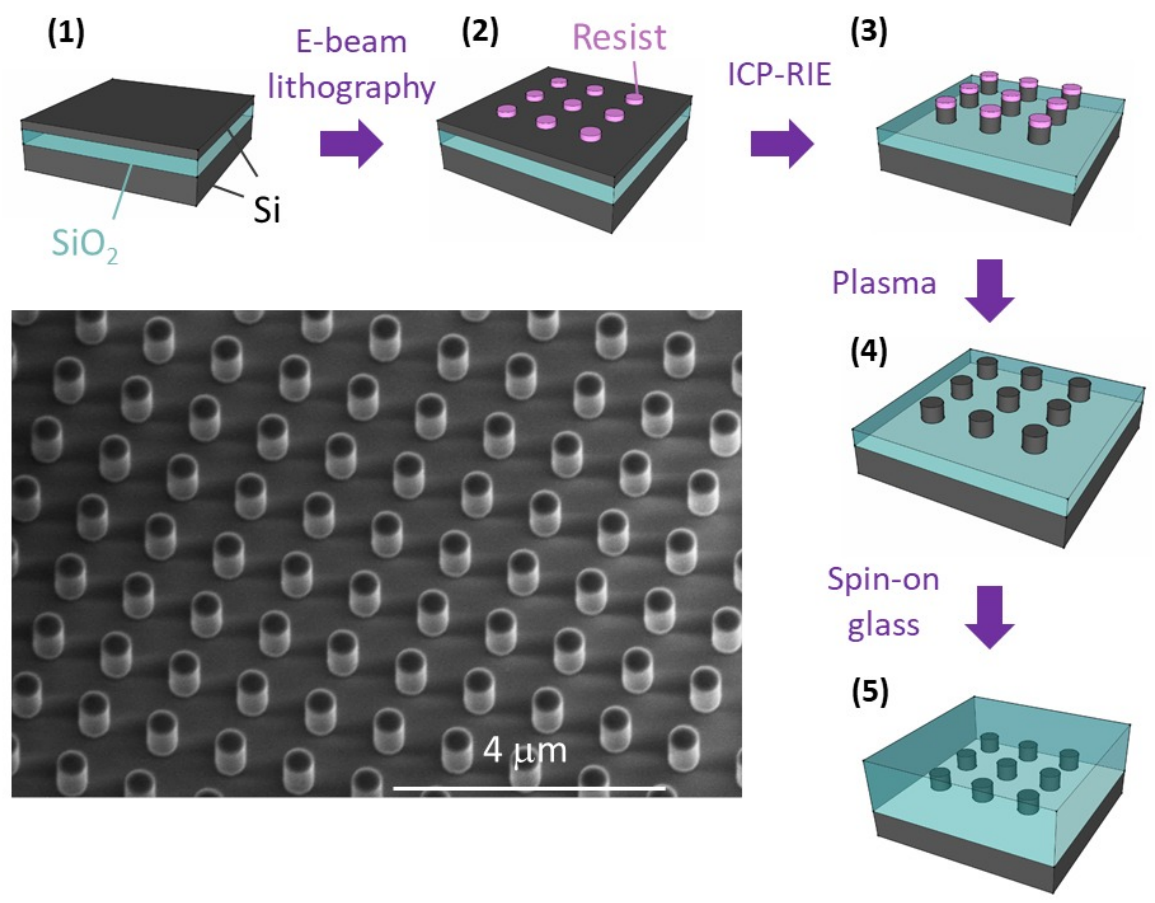

Figure 2. Schematic of fabrication process for nanopillar arrays on SOI and SEM image of array prior to applying spinon glass.

Reflectivity of experimentally fabricated arrays is measured and compared to FDTD simulations (Figure 3). Experimentally determined GMR has a reflectivity of $71 \%$; the peak position of the array in experiment is slightly blueshifted (peak position is located at $1525 \mathrm{~nm}$ against $1542 \mathrm{~nm}$ in simulation). The full-width half max (FWHM) of the measured peak is $24 \mathrm{~nm}$ vs $11 \mathrm{~nm}$ in simulation. These small discrepancies between the experiments and the simulations are likely caused by a slight vertical tapering of the nanopillars characteristic of dry etching processes, size variance among the pillars, or minor differences in the refractive index between the underlying $\mathrm{SiO}_{2}$ and the applied SOG. The narrowness of the resonances allow for the possibility of these fabricated arrays to serve as hyperspectral filters. The amplitude of the experimental reflectivity peak is a factor of 1.36 lower compared with the simulation, likely due to energy lost to the Fabry-Perot interference evidenced by the oscillatory behavior, resulting from the presence of the higher index of the underlying silicon layer relative to that of the oxide layer that encompasses the array. To confirm that 
this effect is, in fact, Fabry-Perot interference we also measured the reflectivity on a region of the as fabricated sample where no silicon array pattern is present. As expected, these measurements revealed fully present oscillations but no GMR.

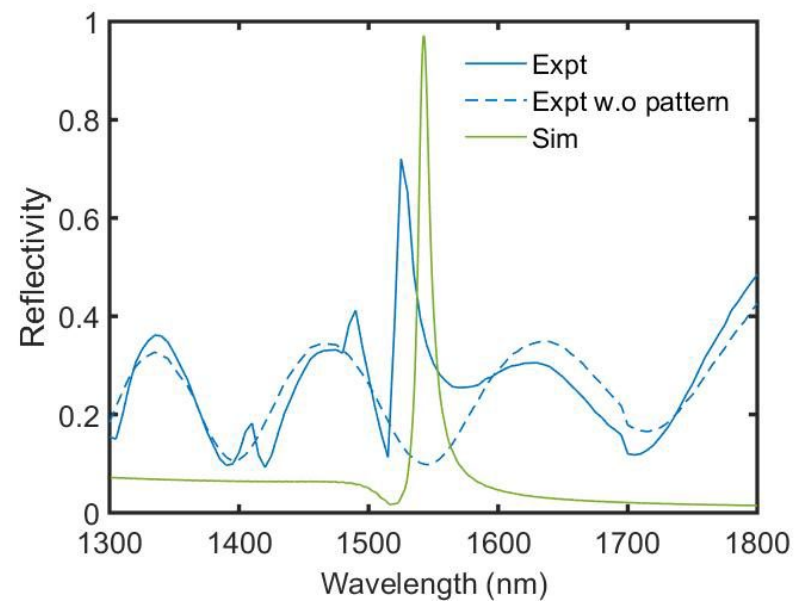

Figure 3. Reflectivity spectrum for a nanopillar array with dimensions $r=210 \mathrm{~nm}, \mathrm{a}=1050 \mathrm{~nm}, \mathrm{~h}=100 \mathrm{~nm}$ for experiment (solid blue line) and simulation (solid green line). Fabry-Perot interference is observed in experiment confirmed by comparison to a spectrum taken on the substrate without a nanopillar array (dashed blue line).

Removing the silicon substrate would allow the Fabry-Perot interference to disappear, thus reducing energy loss and maximizing the amplitude of the GMR. Etching through a $675 \mu \mathrm{m}$-thick substrate layer while maintaining the integrity of the thin oxide containing the array limit the use of wet chemical etchants. Instead, we use ICP-RIE to etch through the entirety of the underlying silicon wafer within the rectangular area shown in Figure 4. . The edges of the sample chip are masked while the center underneath the pattern is etched away. As the etch nears the thin oxide layer, care must be taken as over-etching ruptures the thin oxide layer. The resulting structure is a $3.5 \mu \mathrm{m} \mathrm{SiO}_{2}$ film bounded by silicon supports (Figure 4). The different stiffness between $\mathrm{Si}$ and $\mathrm{SiO}_{2}$ cause the oxide film to wrinkle upon its release from the silicon, which renders measuring specular reflectivity impossible due to significant scattering on the non-flat surface.

(a)

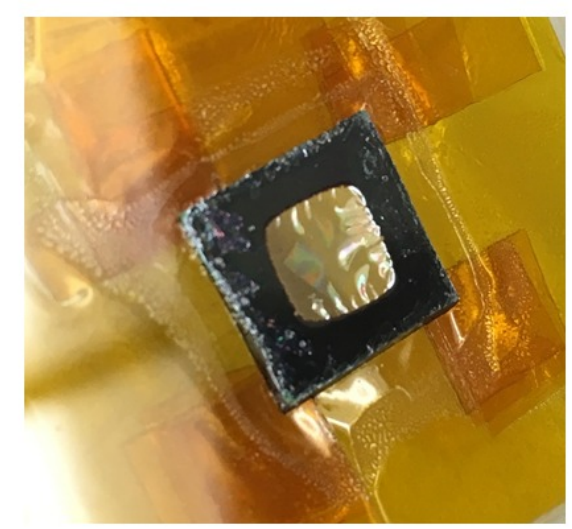

(b)

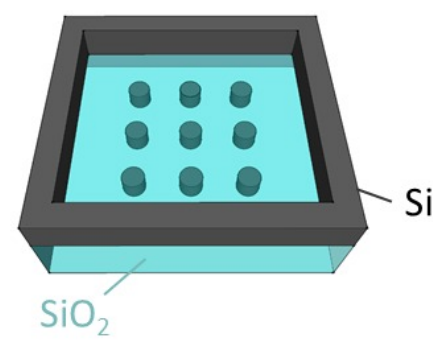

Figure 4. Camera image (a) and schematic drawing (b) of free-standing film containing nanopillar array with silicon supports following etching through the backside silicon substrate. 
To circumvent this challenge we developed an alternative fabrication process that begins with a $\mathrm{SiO}_{2}$ substrate and no underlying silicon and follows a similar fabrication scheme (Figure 5). We first deposit $100 \mathrm{~nm}$ of amorphous silicon (a$\mathrm{Si}$ ) to serve as the device layer via plasma-enhanced chemical vapor deposition (PECVD) which determines the height of our nanopillars. Then we spin-coat $200 \mathrm{~nm}$ of MaN-2403 e-beam resist. To improve substrate conductivity for lithography, we spun a water-soluble sacrificial polymer layer on top of the resist, followed by thermal evaporation of a $10 \mathrm{~nm}$ gold layer. Following e-beam lithography, the sacrificial polymer and gold layers are easily removed in $\mathrm{H}_{2} \mathrm{O}$. After development, the pattern is transferred into the silicon using ICP-RIE etching. To minimize any shifts in the wavelength position of the GMR caused by the slight index differences between the $\mathrm{SiO}_{2}$ substrate and the applied spinon glass, we deposited $400 \mathrm{~nm}$ of $\mathrm{SiO}_{2}$ onto the top of this stack using PECVD. The rough surface of the deposited $\mathrm{SiO}_{2}$ is planarized by applying an additional $500 \mathrm{~nm}$ thick layer of spin-on glass. Reflectivity spectra demonstrating the potential for these nanopillar arrays to serve as spectral filters will be provided in a future publication. Preliminary measurements suggest that such a fabrication process leads to GMRs with greater amplitudes than those fabricated on SOI due to the absence of Fabry-Perot interference.

(1)
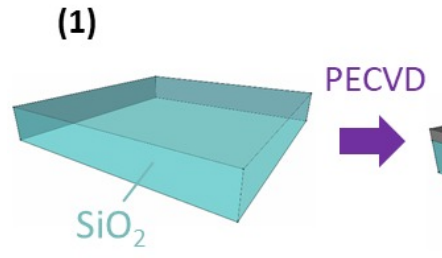

(5)

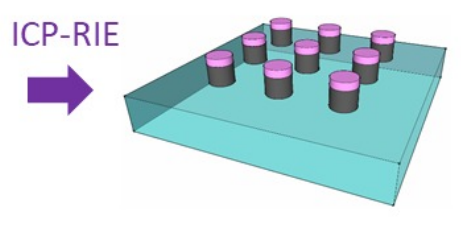

(2)

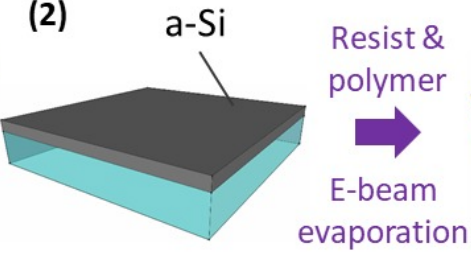

polymer

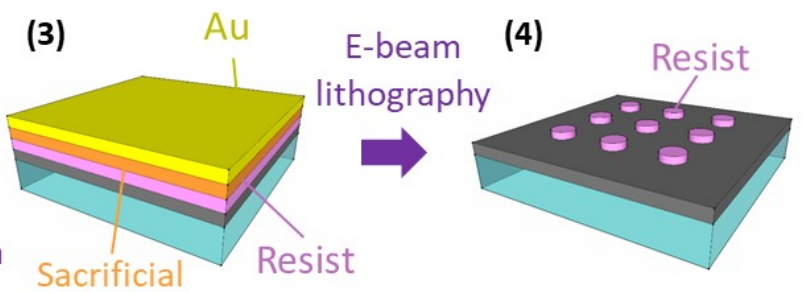

(6)

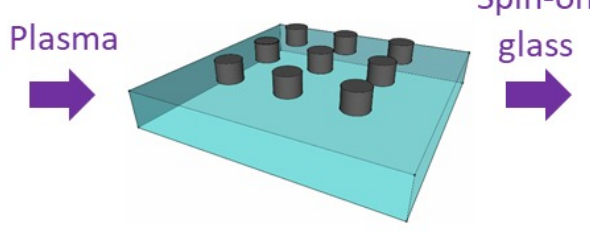

(7)

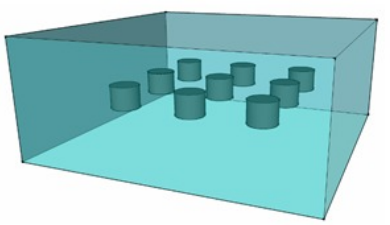

Figure 5. Schematic of fabrication process for nanopillar arrays on a-Si and SEM image of array prior to applying spinon glass.

\section{CONCLUSION}

We demonstrate the fabrication of ultra-thin silicon nanopillar arrays that exhibit near-unity reflectivity resonances that emerges from incident light coupling to a grating vector followed by light reradiation in the absence of any guided modes within the array. The GMRs exhibited by these arrays are easily tunable through parametric variation of the nanopillar radius, height, and array periodicity. For arrays fabricated on SOI substrates, experimentally obtained GMRs were in agreement with FDTD simulations, though Fabry-Perot interference was present as a result of the underlying silicon layer. Efforts to eliminate this interference resulted in scattering of the incident beam, preventing successful reflectivity measurements without the silicon substrate. To overcome this challenge, we propose a fabrication route to create nanopillar arrays without the underlying silicon by using a glass substrate. Preliminary measurements suggest GMRs with amplitudes greater than those measured here experimentally on SOI due to the absence of Fabry-Perot interference. With the FWHM ranging from $<10 \mathrm{~nm}$ to $\sim 30 \mathrm{~nm}$ and near-unity amplitude that are highly controllable, these arrays can easily be manipulated through fabrication to create the contiguous spectral bands required for a hyperspectral imaging system. 


\section{REFERENCES}

[1] Marshall, S., Kelman, T., Qiao, T., Murray, P. and Zabalza, J., "HYPERSPECTRAL IMAGING FOR FOOD APPLICATIONS," Signal Process. Conf., 2854-2858 (2015).

[2] Taylor, P., Feng, Y., Sun, D., Feng, Y. and Sun, D., "Application of Hyperspectral Imaging in Food Safety Inspection and Control : A Review Application of Hyperspectral Imaging in Food Safety Inspection and Control : A Review," Crit. Rev. Food Sci. Nutr. 52(11), 1039-1058 (2012).

[3] Yuen, P. W. T. and Richardson, M., "An introduction to hyperspectral imaging and its application for security , surveillance and target acquisition," Imaging Sci. J. 58, 241-254 (2010).

[4] Leifer, I., Lehr, W. J., Simecek-beatty, D., Bradley, E., Clark, R., Dennison, P., Hu, Y., Matheson, S., Jones, C. E., Holt, B., Reif, M., Roberts, D. A., Svejkovsky, J., Swayze, G. and Wozencraft, J., "Remote Sensing of Environment State of the art satellite and airborne marine oil spill remote sensing : Application to the BP Deepwater Horizon oil spill," Remote Sens. Environ. 124, 185-209 (2012).

[5] Slonecker, T., Fisher, G. B., Aiello, D. P. and Haack, B., "Visible and Infrared Remote Imaging of Hazardous Waste : A," Remote Sens. 2, 2474-2508 (2010).

[6] Jang, W., Ku, Z., Jeon, J., Kim, J. O., Lee, S. J., Park, J., Noyola, M. J. and Urbas, A., "Experimental Demonstration of Adaptive Infrared Multispectral Imaging using Plasmonic Filter Array," Sci. Rep. 6, 1-9 (2016).

[7] Jang, W., Ku, Z., Urbas, A., Derov, J. and Noyola, M. J., "Plasmonic Superpixel Sensor for Compressive Spectral Sensing," IEEE Trans. Geosci. Remote Sens. 53(6), 3471-3480 (2015).

[8] Vaughan, R. G., Calvin, W. M. and Taranik, J. V., "SEBASS hyperspectral thermal infrared data : surface emissivity measurement and mineral mapping," Remote Sens. Environ. 85, 48-63 (2003).

[9] Luceya, P. G., Williamsa, T., Mignarda, M., Juliana, J., Kokobuna, D., Allena, G., Hamptona, D., Schaff, W., Schlangenb, M., Winterb, E. M., Kendalic, W., Stockerc, A., Hortona, K. and Bowmand, A. P., "AHI : An airborne long wave infrared hyperspectral imager," Proc. SPIE 3431(July), 36-43 (1998).

[10] Hackwell, J. A., Warren, D. W., Bongiovi, R. P., Hansel, S. J., Hayhurst, T. L., Dan, J., Sivjee, M. G. and Skinner, J. W., "LWIR/MWIR Imaging Hyperspectral Sensor for Airborne and Ground-Based Remote Sensing," Proc. SPIE 2819(1), 102-107 (1996).

[11] Wang, S. S., Magnusson, R., Bagby, J. S. and Moharam, M. G., "Guided-mode resonances in planar dielectriclayer diffraction gratings," J. Opt. Soc. Am. A 7(8), 1470 (1990).

[12] Wang, S. S. and Magnusson, R., "Theory and applications of guided-mode resonance filters.," Appl. Opt. 32(14), 2606-2613 (1993).

[13] Henry, M. D., "Icp Etching of Silicon for Micro and Nanoscale Devices" (2010). 